\section{Highly polymorphic RFLP marker D4S130 (CRI-L518) maps to distal $4 \mathrm{q}$}

B.Weiffenbach, J.Dubois and R.Bagley

Collaborative Research, Inc., 13565 Main Street, Waltham, MA 02154, USA

Source/Description: D4S130 was isolated from a genomic lambda charon 4A library (1). Insert size is $10-15 \mathrm{~kb}$.

Polymorphism: D4S130 detects a multi-allele polymorphism with HaeIII digests of human DNA; over 14 alleles have been seen. Variable fragments range in size from 1.0 to $3.5 \mathrm{~kb}$. Constant bands are observed at 2.6, 2.0, 1.3, 0.98, 0.82, and $0.74 \mathrm{~kb}$. The probe detects two systems with TaqI digested human DNA. Polymorphisms are also detected with BglII, EcoRI, MspI, PstI, and RsaI. The Taq and HaeIII polymorphisms are in linkage disequilibrium.

Heterozygosity: Using the parents of $21 \mathrm{CEPH}$ families, the observed heterozygosity for the HaeIII polymorphism was 0.71 and the PIC was 0.69

Frequency: The following frequencies of HaeIII alleles were seen in $18 \mathrm{CEPH}$ parents

\begin{tabular}{|c|c|c|c|c|c|}
\hline Allele & Size (kb) & Frequency & Allele & Size $(k b)$ & Frequency \\
\hline $\mathrm{Cl}$ & 3.1 & $2.8 \%(1 / 36)$ & $\mathrm{C} 8$ & 1.85 & $2.8 \%(1 / 36)$ \\
\hline $\mathrm{C} 2$ & 2.95 & $5.6 \%(2 / 36)$ & $\mathrm{C} 9$ & 1.6 & $11.1 \%(4 / 36)$ \\
\hline C3 & 2.9 & $2.8 \%(1 / 36)$ & $\mathrm{C} 10$ & 1.55 & $5.6 \%(2 / 36)$ \\
\hline C4 & 2.8 & $11.1 \%(4 / 36)$ & $\mathrm{C} 11$ & 1.38 & $8.3 \%(3 / 36)$ \\
\hline C5 & 2.2 & $2.8 \%(1 / 36)$ & $\mathrm{C} 12$ & 1.35 & $11.1 \%(4 / 36)$ \\
\hline C6 & 2.1 & $22 \%(8 / 36)$ & $\mathrm{C} 13$ & 1.26 & $5.6 \%(2 / 36)$ \\
\hline C7 & 2.05 & $2.8 \%(1 / 36)$ & $\mathrm{C} 14$ & 1.2 & $5.6 \%(2 / 36)$ \\
\hline
\end{tabular}

Not Polymorphic For: BamHI and HindIII.

Chromosomal Localization: D4S130 was provisionally assigned to chromosome 22 based on a two-point lod score greater than 3 but less than 4 (2). It was later reassigned to chromosome 4 by a somatic cell hybrid panel (3) but was unlinked to most chromosome 4 markers. We now find that HaeIII RFLP detected by $\mathrm{D} 4 \mathrm{~S} 130$ is tightly linked to distal chromosome $4 \mathrm{q}$ markers D4S139 (4q35), D4S184, and D4S171 using 40 CEPH reference families.

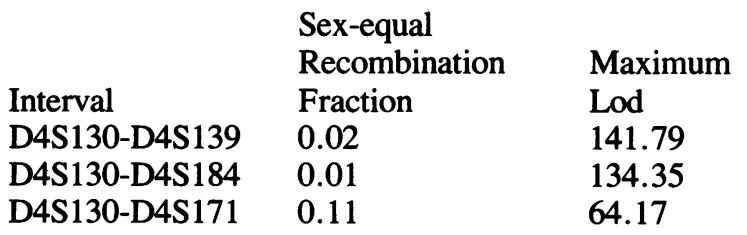

Mendelian Inheritance: Co-dominant inheritance of the HaeIII RFLP was seen in the 36 multigenerational CEPH families.

Probe Availability: D4S130 is available for research use. Contact G. Vovis, Collaborative Research, Inc.

References: 1) Lawn et al. (1978) Cell 15, 1151-1174. 2) DonisKeller et al. (1987) Cell 51, 319-337. 3) Donis-Keller et al. (1989) Cytogenet. Cell Genet. 51, 991-992.

\section{A Pvull polymorphism detected by pEW404 (D17S64) on chromosome 17}

A.M.Lucas, J.M.Vance*, J.M.Stajich and A.D.Roses

Box 2900, Division of Neurology, Duke University Medical Center, Durham, NC 27710, USA

Source and Description: pEW404 (D17S64) is a $1.4 \mathrm{~kb}$ genomic fragment subcloned into plasmid pUC8.

Polymorphism: PvuII detects a two allele polymorphism with fragments of $13.0 \mathrm{~B} 1$ and $7.8 \mathrm{~B} 2 \mathrm{~kb}$.

Frequency: Estimated from 42 unrelated Caucasians

$13.0 \mathrm{~kb}(\mathrm{~B} 1)=.4$

$7.8 \mathrm{~kb}(\mathrm{~B} 2)=.6$

Observed heterozygosity is $\mathbf{0 . 4 3}$

Not Polymorphic For: BamHI, BglI, BglII, EcoRI, EcoRV, HaeIII, HincII, HindIII, HinfI, KpnI, NocI, RsaI, SacI, SstI, StuI, TaqI, XbaI (studied in 6 unrelated individuals).

Chromosomal Location: 17p11.2-17p12.0 (1).

Mendelian Inheritance: Co-dominant segregation was observed in 6 families.

Probe Availability: Available from ATCC.

Other Comments: RFLP was observed under hybridization conditions using preannealing with Human Placental DNA and very stringent washes. Linkage disequilibrium was not observed between the MspI (1) and PVUII polymorphisms.

Acknowledgements: Supported by grants from NINDS \# NS26630, NS01289, and a grant from the Muscular Dystrophy Association. pEW404 was kindly obtained from Dr David Barker.

References: 1) Solomon,E. and Barker,D.F. (1989) Cytogenetics and Cell Genetics 51, 319-337.

* To whom correspondence should be addressed 